\title{
A case of pilocytic astrocytoma requiring tumor resection during pregnancy
}

\author{
TORU UMEHARA ${ }^{1}$, YOSHIKO OKITA ${ }^{1}$, MASAHIRO NONAKA ${ }^{1}$, YONEHIRO KANEMURA ${ }^{1,2}$, \\ YOSHINORI KODAMA $^{3}$, MASAYUKI MANO ${ }^{3}$, SHIN NAKAJIMA ${ }^{1}$ and TOSHIYUKI FUJINAKA ${ }^{1}$ \\ ${ }^{1}$ Department of Neurosurgery; ${ }^{2}$ Division of Regenerative Medicine, Institute for Clinical Research; \\ ${ }^{3}$ Department of Central Laboratory and Surgical Pathology, Osaka National Hospital, \\ National Hospital Organization, Osaka 540-0006, Japan
}

Received September 16, 2015; Accepted February 3, 2016

DOI: $10.3892 / \mathrm{mco} .2016 .773$

\begin{abstract}
Low-grade glioma (LGG) is often encountered in relatively young individuals, including women of childbearing age. Notably, case series describing pregnant women with LGG have been reported in the literature. The present study reported a case of pilocytic astrocytoma (PA) requiring tumor resection during pregnancy. The patient had a history of $\gamma$-knife radiotherapy for a brainstem tumor 17 years previously. The histological diagnosis was unclear. The tumor had remained stable following radiosurgery for 17 years, including during her first pregnancy. However, rapid tumor growth around the fourth ventricle occurred at week 25 of her second pregnancy. Therefore, an urgent tumor resection was performed despite the pregnancy. Partial resection was performed since the tumor had infiltrated the brainstem. The histological diagnosis was PA. The residual tumor volume decreased gradually following the delivery. Immunostaining of the tumor for the expression of progesterone receptor revealed focal staining. It is possible that reproductive factors, including specific hormonal changes during pregnancy, affected the tumor growth. The present study described this rare case of PA, which exhibited rapid growth and required urgent surgery during pregnancy.
\end{abstract}

\section{Introduction}

Low-grade glioma (LGG) is often encountered in relatively young individuals, including women of childbearing age. Advances in the therapeutic management of LGG during the last decade and improvements in its prognosis are expected to assist young women with LGG in becoming pregnant. A number of case series are found in the literature describing

Correspondence to: Dr Yoshiko Okita, Department of Neurosurgery, Osaka National Hospital, National Hospital Organization, 14-1-2 Hoenzaka, Chuo-ku, Osaka-shi, Osaka 540-0006, Japan

E-mail: yokita4246@gmail.com

Key words: pregnancy, pilocytic astrocy toma, tumor growth pregnant women with LGG, either detected prior to pregnancy or newly diagnosed during pregnancy (1-10). In either case, its management is a particularly challenging situation since i) anti-tumor therapy is required, although the impact on the fetus or the pregnancy would require consideration or ii) the pregnancy would have to be discontinued, depending on the severity of the disease and the gestational age (10). Once a pregnancy with LGG is identified, patients, families, and neurosurgeons must recognize that they will encounter numerous difficult decisions at each stage of the pregnancy.

The association between the behavior of the glioma and the pregnancy remains to be elucidated. Several previous studies have reported that the velocity of the diametric expansion of LGG increases during pregnancy (8) and that this acceleration may depend on multiple factors, including hormonal factors (11), growth factors (12), and hemodynamic changes $(1,13)$, associated with pregnancy.

Pilocytic astrocytoma (PA) is typically a slow-growing astrocytoma and is therefore, considered the 'most benign' of all the astrocytomas. It occurs predominantly in childhood and adolescence (14). Growth of PA during pregnancy is rarely reported. The present study reported a case in which PA growth occurred and urgent resection was required during pregnancy. The behavior of the glioma is focussed on during the pregnancy.

\section{Case report}

A 30-year-old pregnant woman with a history of a brainstem tumor, which developed around the dorsal midbrain, spreading from the third to fourth ventricle was included in the present study. Although the tumor had remained pathologically undiagnosed, despite a biopsy at a previous medical institution, the patient received $\gamma$-knife therapy and ventriculoperitoneal shunt (VPS) placement at 13 years of age. The tumor remained stable following radiosurgery and the patient had undergone several VPS revisions for shunt malfunction during adolescence.

At 29-years-old, the patient became pregnant for the first time. Owing to her history of the brainstem tumor and VPS placement, she was referred to our institution at that time. She developed an eye movement disorder during the third trimester of the pregnancy, which suggested shunt malfunction 

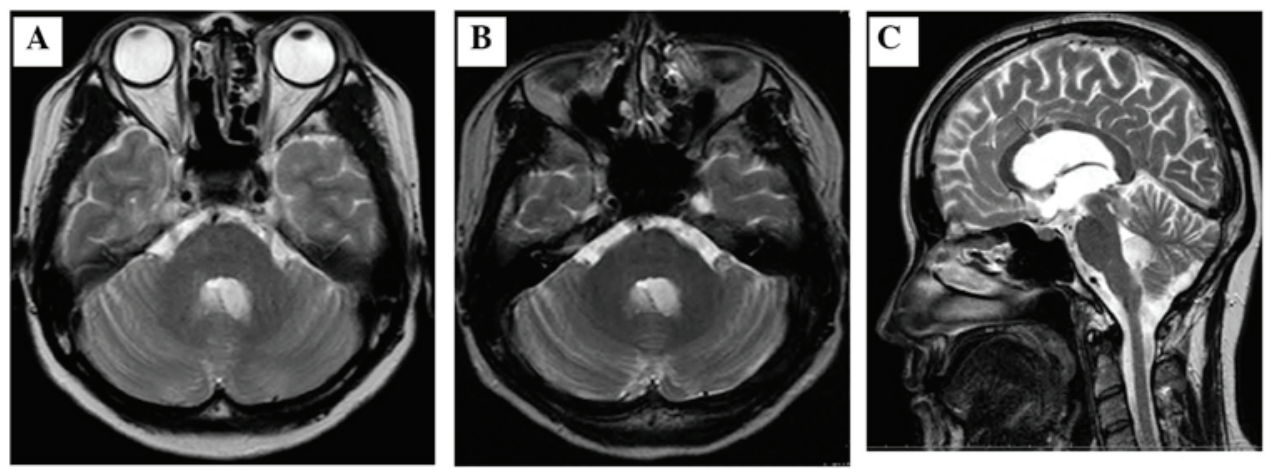

Figure 1. Axial and sagittal T2-weighted magnetic resonance imaging scans obtained during the first pregnancy and puerperium. The lesion in the fourth ventricle displays high intensity on the T2-weighted image. (A) The axial image was obtained at 26 weeks of gestation during the first pregnancy when the patient was referred to our institution. (B and C) Axial and sagittal images were obtained shortly following the first delivery. The images reveal no significant changes in tumor behavior during the first pregnancy.

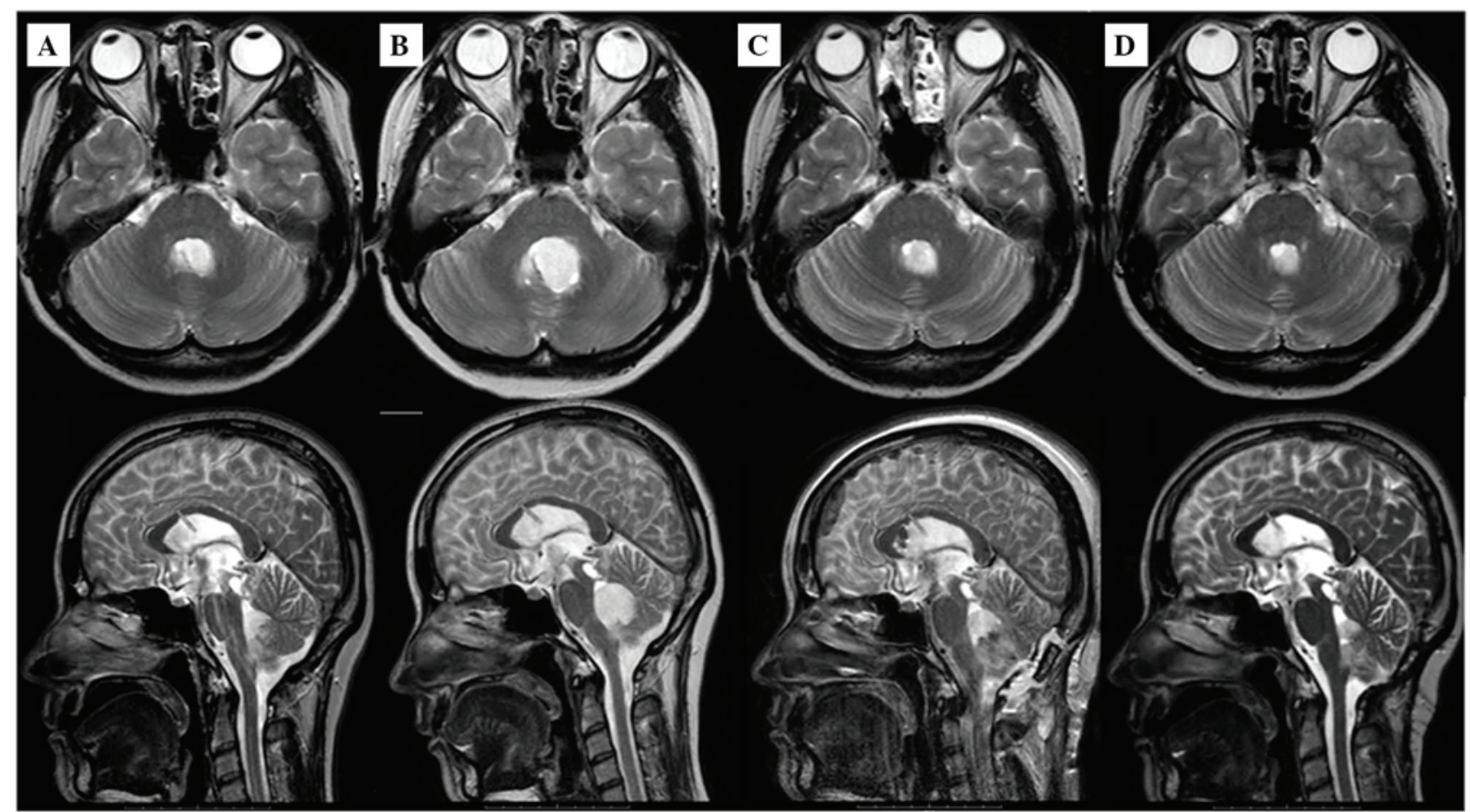

Figure 2. Axial and sagittal T2-weighted magnetic resonance imaging scans obtained during the patient's second pregnancy, following neurosurgery and after delivery. (A) Images were obtained at 5 weeks of gestation during the patient's second pregnancy. (B) Images at 25 weeks of gestation during the patient's second pregnancy indicated rapid tumor growth in the second trimester. The tumor volume increased 3 -fold during the first 5 months of the second pregnancy. (C) Images were obtained following the operation at 26 weeks of gestation where $\sim 70 \%$ of the tumor was resected. (D) Images were obtained 7 months after the second delivery. The tumor volume decreased by up to $50 \%$ compared with the post-operative images without any tumor treatment.

due to the increased intra-abdominal pressure caused by the gravid uterus. The patient underwent a cesarean delivery (CS) and an external ventricle drain at 36 weeks of gestation under epidural anesthesia. Due to the patient's desire to bear more children in the future, an endoscopic third ventriculostomy (ETV) and to withdraw from VPS placement was planned. Therefore, two weeks following delivery, the patient underwent an ETV and an endoscopic biopsy of the residual tumor in the third ventricle. No pathological confirmation of disease was detected across several specimens. The ETV was highly effective and the VPS was removed, as planned. The post-operative course was uneventful and the patient was discharged from hospital with a healthy son. Notably, no significant changes in the behavior of the brain tumor was observed during the first pregnancy (Fig. 1).

One year later, the patient became pregnant for the second time, the patient was 30 -years-old. The tumor remained stable during the first trimester (Fig. 2A). However, she presented with nausea and headache 5 months into the pregnancy. Magnetic resonance imaging (MRI) of the head revealed rapid tumor growth in the fourth ventricle and cerebellum (Fig. 2B), which necessitated an urgent resection. At the 25-week gestation point, abortion is prohibited. As fetal maturity was not sufficient to proceed with the delivery, tumor resection was performed with the patient under general anesthesia, thereby allowing the pregnancy to continue. As the tumor had infiltrated the dorsal 


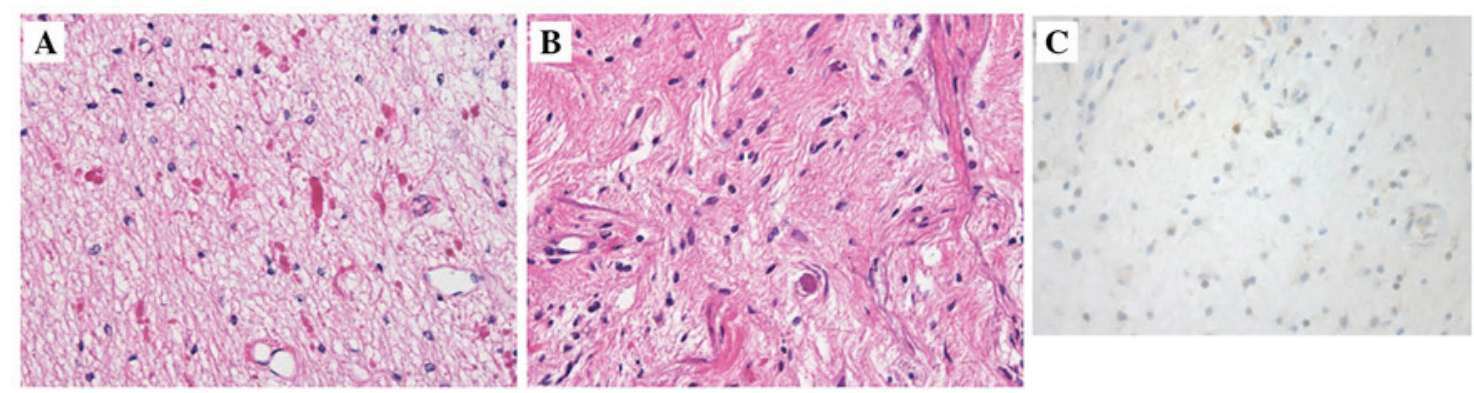

Figure 3. Pathological findings. (A) Hematoxylin and eosin staining demonstrated moderate cellularity with Rosenthal fibers and eosinophilic granular bodies. (B) A micrograph revealed growth of compacted bipolar or unipolar cells with elongated hair-like processes. (C) Immunostaining for the expression of the progesterone receptor revealed focal staining in the tumor.

brainstem, a partial resection was performed (Fig. 2C). The fetal heart rate was monitored and a good fetal condition was maintained during the operation. Histological examination revealed a World Health Organization grade I PA, with moderate cellularity exhibited by a number of compacted bipolar cells with Rosenthal fibers and eosinophilic granular bodies (Fig. 3A and B). Immunostaining for progesterone receptor expression revealed focal staining in the tumor (Fig. 3C). The patient had an uneventful recovery and was discharged 2 weeks after the operation. At week 37 of the pregnancy, the patient delivered a healthy daughter by CS. No complementary treatment was administered. The patient exhibited no relapse of tumor growth during the perinatal period. By contrast, the follow-up MRIs revealed a significantly decreased tumor volume by up to $\sim 50 \%$ in just 7 months following delivery (Figs. 2D and 4).

\section{Discussion}

Glioma during pregnancy is a rare occurrence. The incidence of malignant brain tumors in pregnancy is 3.6 per million live births (15), and its management is often quite difficult. The treatment of glioma during pregnancy is complex as it may be associated with adverse effects on the fetus. If fetal pulmonary maturity has been attained, delivery prior to neurosurgery is preferable (16). Prompt therapy in parallel with the pregnancy appears to be more actively required in patients with high-grade glioma (HGG) or newly diagnosed symptomatic LGG during pregnancy compared with in patients with known LGG (kLGG) prior to pregnancy $(2-6,9)$. LGG is a slow-growing tumor and the majority of pregnant women with kLGG give birth as planned and are less likely to receive any urgent therapy during pregnancy $(7,8,10)$. Therefore, operative treatment during pregnancy for kLGG is extremely rare. However, there are, of course, exceptions to the clinical course of the disease. In a case series by Zwinkels et al (10), two pregnant women with $\mathrm{kLGG}$ received therapeutic abortions during the first trimester as a result of rapid tumor progression and subsequent tumor resection was performed (10). Daras et al (17) reported three cases of kLGG, which demonstrated neurological deterioration during pregnancy or puerperium due to malignant transformation of LGG to HGG. Of these, one patient underwent a tumor resection at 24 weeks of gestation during her pregnancy and the other two received radiation and chemotherapy following the gestation period. In the present case, no option

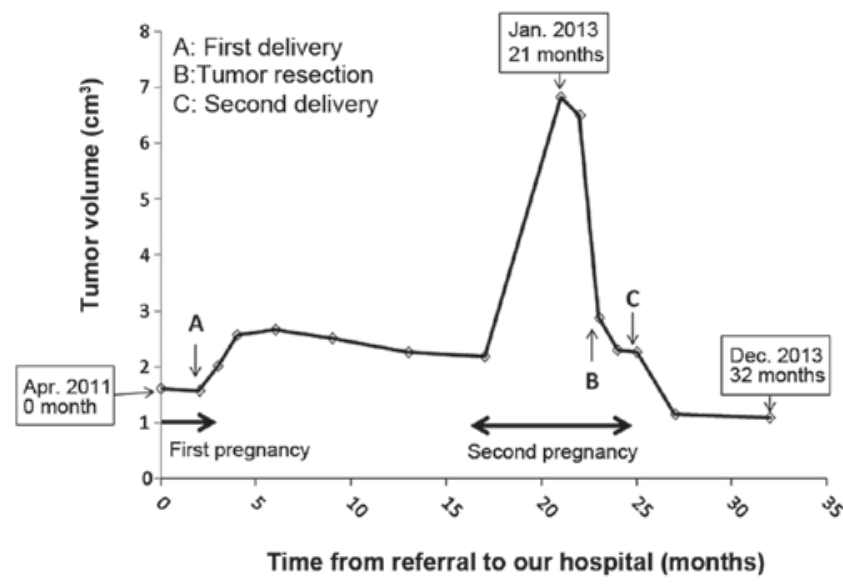

Figure 4. Changes in the tumor volume on repeated magnetic resonance imaging scans between the first pregnancy until after the second pregnancy. The tumor volume was calculated from the images using Brain SCAN software. The schematic demonstrated the rapid increase in the tumor volume during the second pregnancy, the decrease following the partial resection, and the further spontaneous decrease following delivery.

was available other than urgent surgical treatment and the continuation of the pregnancy as the patient presented with neurological deterioration associated with tumor growth and fetal maturity had not been achieved.

PA is a circumscribed, slow-growing, benign astrocytoma that occurs predominantly in childhood and adolescence (14). Despite an extensive literature search, only one report of a PA associated with pregnancy exists and it had required tumor resection (6). The PA in this case was newly diagnosed during pregnancy and surgery was performed shortly after delivery (6). By contrast, the PA in our case was observed carefully for 17 years and the tumor volume remained stable until the patient's second pregnancy. Despite careful observation, rapid tumor growth occurred during the second pregnancy and tumor resection was performed during the pregnancy. Although tumor growth of PA during pregnancy is extremely rare, neurosurgeons should maintain careful observation when young women with PAs are considering becoming pregnant.

The mechanism by which pregnancy affects glioma has remained unclear. Pregnancy leads to major changes in the levels of pregnancy hormones. Progesterone levels are dramatically elevated during a pregnancy, particularly during the later stages (18). Gonzalez-Aguero et al (11) reported that progesterone induces cell growth in human astrocytomas (11). 
Khalid et al (19) suggested that progesterone receptors may be involved in the growth of astrocytic tumors (19). In the present case, immunostaining for the expression of progesterone receptor revealed focal staining in the tumor. Symptomatic appearance in our patient began at 5 months into the pregnancy, and the tumor volume significantly decreased following delivery. It is possible that changes in progesterone levels induced progesterone receptor activation and affected tumor growth and regression in our patient.

In conclusion, the present study reported a case of PA, which exhibited progression and required urgent neurosurgical treatment during the patient's second pregnancy. While this was an extremely rare event, the changes in progesterone levels during pregnancy may have induced progesterone receptor activation and affected tumor growth. Neurosurgeons and obstetricians should recognize the possibility of tumor progression during each pregnancy and the necessity of close neurological follow-up with repeated MRIs and obstetrical monitoring, even if the histological type is not typically malignant and no observable tumor growth occurs during previous pregnancies. The present study is an important clinical case of a brain tumor during pregnancy, which gives cause for alarm.

\section{References}

1. Blumenthal DT, Parreño MG, Batten J and Chamberlain MC: Management of malignant gliomas during pregnancy: A case series. Cancer 113: 3349-3354, 2008.

2. Cohen-Gadol AA, Friedman JA, Friedman JD, Tubbs RS, Munis JR and Meyer FB: Neurosurgical management of intracranial lesions in the pregnant patient: A 36-year institutional experience and review of the literature. J Neurosurg 111: $1150-1157,2009$.

3. Isla A, Alvarez F, Gonzalez A, García-Grande A,Perez-Alvarez M and García-Blazquez M: Brain tumor and pregnancy. Obstet Gynecol 89: 19-23, 1997.

4. Johnson N, Sermer M, Lausman A and Maxwell C: Obstetric outcomes of women with intracranial neoplasms. Int J Gynaecol Obstet 105: 56-59, 2009.

5. Lynch JC, Gouvêa F, Emmerich JC, Kokinovrachos G, Pereira C, Welling $\mathrm{L}$ and Kislanov $\mathrm{S}$ : Management strategy for brain tumour diagnosed during pregnancy. Br J Neurosurg 25: 225-230, 2011.

6. Nishio S, Morioka T, Suzuki S, Takeshita I, Ikezaki K, Fukui M and Nakano H: Primary brain tumours manifesting during pregnancy: Presentation of six cases and a review of the literature. J Clin Neurosci 3: 334-337, 1996.
7. Pallud J, Duffau H, Razak RA, Barbarino-Monnier P, Capelle L, Fontaine D, Frenay M, Guillet-May F, Mandonnet E and Taillandier L: Influence of pregnancy in the behavior of diffuse gliomas: Clinical cases of a French glioma study group. J Neurol 256: 2014-2020, 2009.

8. Pallud J, Mandonnet E, Deroulers C, Fontaine D, Badoual M, Capelle L, Guillet-May F, Page P, Peruzzi P, Jouanneau E, et al: Pregnancy increases the growth rates of World Health Organization grade II gliomas. Ann Neurol 67: 398-404, 2010.

9. Sneed PK, Albright NW, Wara WM, Prados MD and Wilson CB: Fetal dose estimates for radiotherapy of brain tumors during pregnancy. Int J Radiat Oncol Biol Phys 32: 823-830, 1995.

10. Zwinkels H, Dörr J, Kloet F, Taphoorn MJ and Vecht CJ: Pregnancy in women with gliomas: A case-series and review of the literature. J Neurooncol 115: 293-301, 2013.

11. González-Agüero G, Gutiérrez AA, González-Espinosa D, Solano JD, Morales R, González-Arenas A, Cabrera-Muñoz E and Camacho-Arroyo I: Progesterone effects on cell growth of U373 and D54 human astrocytoma cell lines. Endocrine 32: 129-135, 2007.

12. Schlenska-Lange A, Knüpfer H, Lange TJ, Kiess W and Knüpfer M: Cell proliferation and migration in glioblastoma multiforme cell lines are influenced by insulin-like growth factor I in vitro. Anticancer Res 28: 1055-1060, 2008.

13. Ducray F, Colin P, Cartalat-Carel S, Pelissou-Guyotat I, Mahla K, Audra P, Gaucherand P, Honnorat J and Trouillas P: Management of malignant gliomas diagnosed during pregnancy. Rev Neurol (Paris) 162: 322-329, 2006 (In French).

14. Huang H, Hara A, Homma T, Yonekawa Y and Ohgaki H: Altered expression of immune defense genes in pilocytic astrocytomas. J Neuropathol Exp Neurol 64: 891-901, 2005.

15. Haas JF, Jänisch W and Staneczek W: Newly diagnosed primary intracranial neoplasms in pregnant women: A population-based assessment. J Neurol Neurosurg Psychiatry 49: 874-880, 1986.

16. Tewari KS, Cappuccini F, Asrat T, Flamm BL, Carpenter SE, Disaia PJ and Quilligan EJ: Obstetric emergencies precipitated by malignant brain tumors. Am J Obstet Gynecol 182: 1215-1221, 2000.

17. Daras M, Cone C and Peters KB: Tumor progression and transformation of low-grade glial tumors associated with pregnancy. J Neurooncol 116: 113-117, 2014.

18. Li R, Scanlon KS and Serdula MK: The validity and reliability of maternal recall of breastfeeding practice. Nutr Rev 63: 103-110, 2005.

19. Khalid H, Shibata S, Kishikawa M, Yasunaga A, Iseki M and Hiura T: Immunohistochemical analysis of progesterone receptor and Ki-67 labeling index in astrocytic tumors. Cancer 80: 2133-2140, 1997. 